\title{
A Multiscale Virtual Reality Approach to Chemical Experiments
}

\author{
Antonio Riganelli ${ }^{1}$, Osvaldo Gervasi ${ }^{2}$, Antonio Laganà ${ }^{1}$, and \\ Margarita Albertì ${ }^{3}$ \\ 1 Department of Chemistry, University of Perugia, Via Elce di Sotto, 8, \\ 06123 Perugia, Italy \\ \{lag, auto\}@dyn.unipg.it \\ 2 Department of Mathematics and Informatics, University of Perugia, \\ Via Vanvitelli, 1, 06123 Perugia, Italy \\ osvaldo@unipg.it \\ http://ogervasi.unipg.it \\ 3 Department of Physical Chemistry, University of Barcelona, \\ Marti i Franques, 1, 08028 Barcelona, Spain
}

\begin{abstract}
A multiscale virtual reality approach to chemical experiments has been implemented using VRML, CML and Java technologies. Three case studies of increasing difficulty are examined to the end of dealing with different combinations of human and molecular scale virtual reality approaches.
\end{abstract}

\section{Introduction}

Among natural sciences Chemistry is by definition a phenomenological discipline rationalized in terms of an invisible reality (atoms and molecules) whose properties and rules are based on the first principles of physics. This has fostered, in the past, the development of chemical models and, in recent times, of chemical simulations. More recently, thanks to the development of high performance concurrent platforms, the role of simulations in chemical investigation has rapidly increased and research has moved from simple to complex systems and from abstract to realistic models embracing full dimensional theoretical treatments which include both human (meter) and molecular (nanometer) scales. However, most often, the related computational apparatus is so heavy and the feedback so poor that the insight obtained from a simulation is very limited even if supported by traditional post-processing visual representations[1].

Analogues of natural phenomena and visual representations have always played a crucial role in the development of science, in general, and of chemistry, in particular $[2,3]$. The most recent step of this ongoing process of making analogues of the real world is the use of virtual reality, VR (see for example refs. [4-7]), at both meter and nanometer level. The present computational capacity (in particular, massively parallel and grid computing) makes it possible, in fact, 
to link the heavy number crunching activity of a simulation with realistic sensorial perceptions of the user at both levels in order to enhance his/her insight and intuition. To this end, the virtual experiment (human virtual reality, HVR) and the simulated interaction between atoms and molecules (molecular virtual reality, MVR) can be combined together in a multiscale approach.

Along this line we are building some virtual chemical experiments three of which are described in this paper. In Section 2 we analyze the computational tools used and the objects defined for assembling the HVR and the MVR environments. In Section 3 we describe in some detail the HVR components of the three chemical experiments. In Section 4 an MVR component of a virtual reality simulation of the proposed experiments is discussed.

\section{Tools and Objects for the HVR and MVR}

Contrary to conventional visualization paradigms, the virtual reality ones when applied to a chemical laboratory (see for examplet Ref.[8] for a multimedia product used for teaching laboratory practices) allow the user to visualize, manipulate and interact with the computer generated structures and materials of the experimental setup being used and of the processes being considered. This is obtained by introducing some elements of immersive VR. These elements make the user an active component of the virtual scene especially if supported by suitable immersive devices like the Head Mounted Display (HMD), the Tracking Devices and/or the Data Gloves.

In the work reported in this paper VR applications are implemented using a Window of World (WoW)[9] approach based on the following tools:

VRML:[10,11] to represent the Molecular Virtual World and to enable its navigation using suitable VRML Web browsers[12]. A migration to $\mathrm{X} 3 \mathrm{D}[13]$ is planned for the near future.

Java:[14] to automate the generation of the necessary VRML code from the data produced by the computational engines used in the simulation and to assemble a dynamical representation of the Molecular Virtual World. Along this line a set of specific Java classes has been created to associate the proper chemical and visual properties (like size and color) to each atom. The simulation provides a description of the system considered in terms of the number of the constituting atoms and bonds. For each atom the chemical symbol, the spatial coordinates of each considered arrangement, the number and the type of bonds are specified. The Java program recognizes the atoms from the chemical symbol, extracts its chemical and visual properties and generates the VRML code for the dynamical rendering of the Molecular Virtual World.

CML:[15] to deal properly, in conjunction with XML[16], with the representation of the physical and chemical properties of the intervening atoms and molecules. 
Using the above mentioned tools a typical HVR chemical laboratory environment was built. A key component of such a virtual chemical laboratory is given in Fig. 1 where a laboratory bench provided with a fume-hood is shown. In the figure also some laboratory basic tools are shown. In this virtual laboratory the user can walk-by, use the various components and perform the implemented experiments (as described in Section 3).

\section{The Chemical Experiments}

The up-to-date implemented experiments are the following.

The first experiment deals with the measurement of the dependence of the volume of a gas from the pressure at constant temperature (Boyle law) [17]. The related HVR environment is the one in which the user adjusts the height of a mercury container connected by a rubber pipe to an air filled burette. By placing the container at various heights one obtains an imbalance in the observed levels of mercury (and therefore a larger or a smaller pressure) as well as a change in the air volume in the burette. By plotting the measured gas volume as a function of the pressure exerted on it, one can work out the proportionality constant that is related to the Boltzmann constant $k_{B}[18]$. The rationalization of the experiment at MVR level is given by a molecular dynamics application (the same used also for the other experiments and illustrated in Section 4). Using that application the behaviour of the gas + liquid $\mathrm{Hg}$ system is mimicked.

The second experiment deals with flame spectroscopy $[19,20]$. The colour of the flame depends on the emission spectrum of the substance added to it. In the HVR environment the user first cleans a wire loop of platinum or nickelchromium by dipping it into a vessel containing hydrochloric or nitric acid and then he/she immerges it into the powder or the solution of an ionic (metal) salt. The loop is finally placed in the clear or blue part of the flame and the resulting colour is observed. A spectroscopic data base allows the individualization of the colours associated with a given element and the processes occurring inside the flame is simulated using a VMR component based on molecular dynamics.

The third experiment is concerned with an expansion jet of a given gas. In the HVR environment a gas is allowed to flow through a valve from a high pressure chamber into a low pressure region. This generates an expansion regime within which several physical and chemical processes can occur including non reactive energy transfer, reactive processes, recombinations, electronic transitions, etc.. In this case too, the VMR component is simulated using a set of molecular dynamics programs.

Accordingly, three specific virtual experimental apparatuses were built. These are:

a) A mercury containing container connected to a burette through a rubber pipe;

b) A platinum wire loop;

c) A high pressure chamber connected to an expansion vessel through an air valve. 


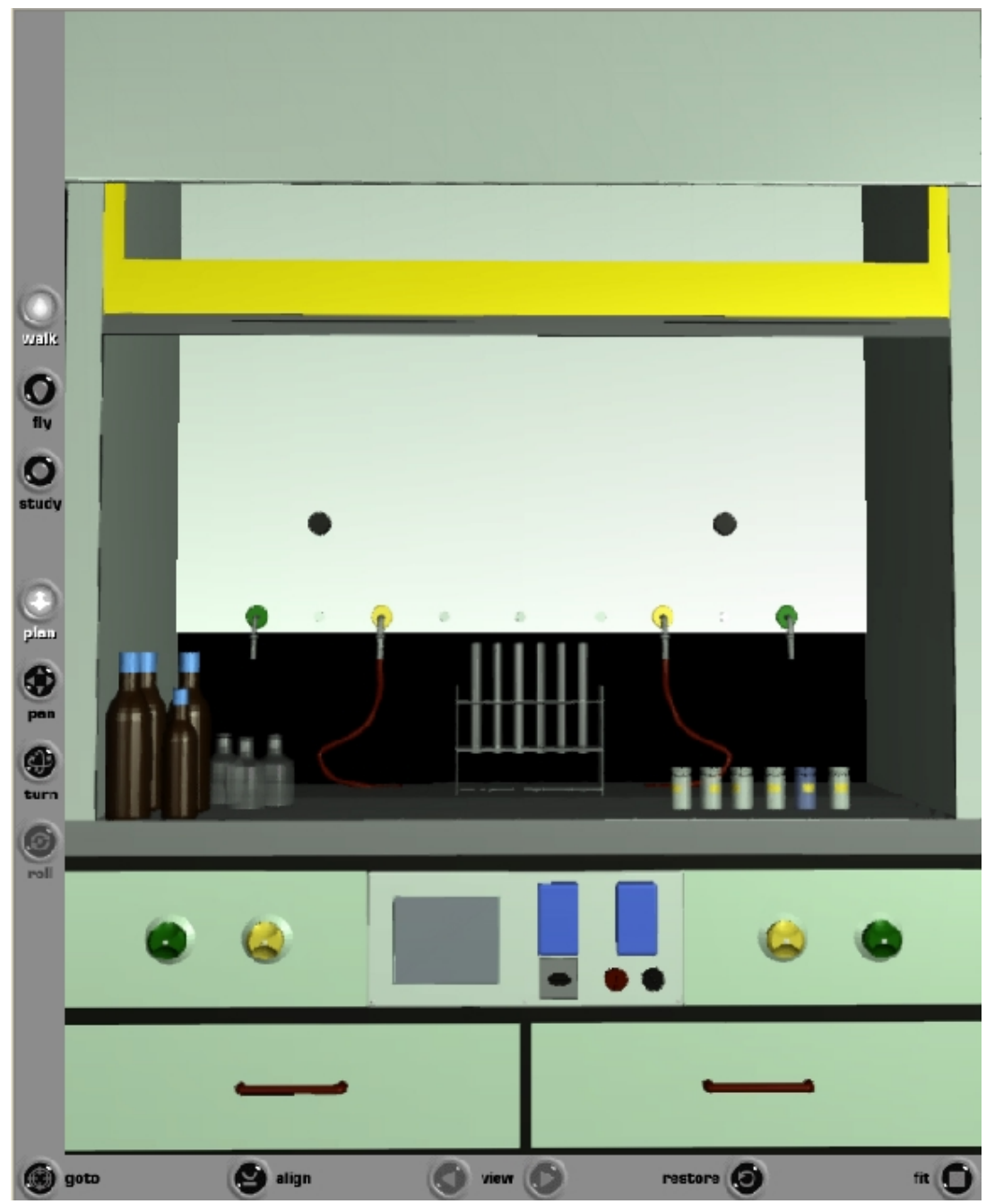

Fig. 1. An HVR illustration of a laboratory bench provided by a fume-hood and some basic tools 


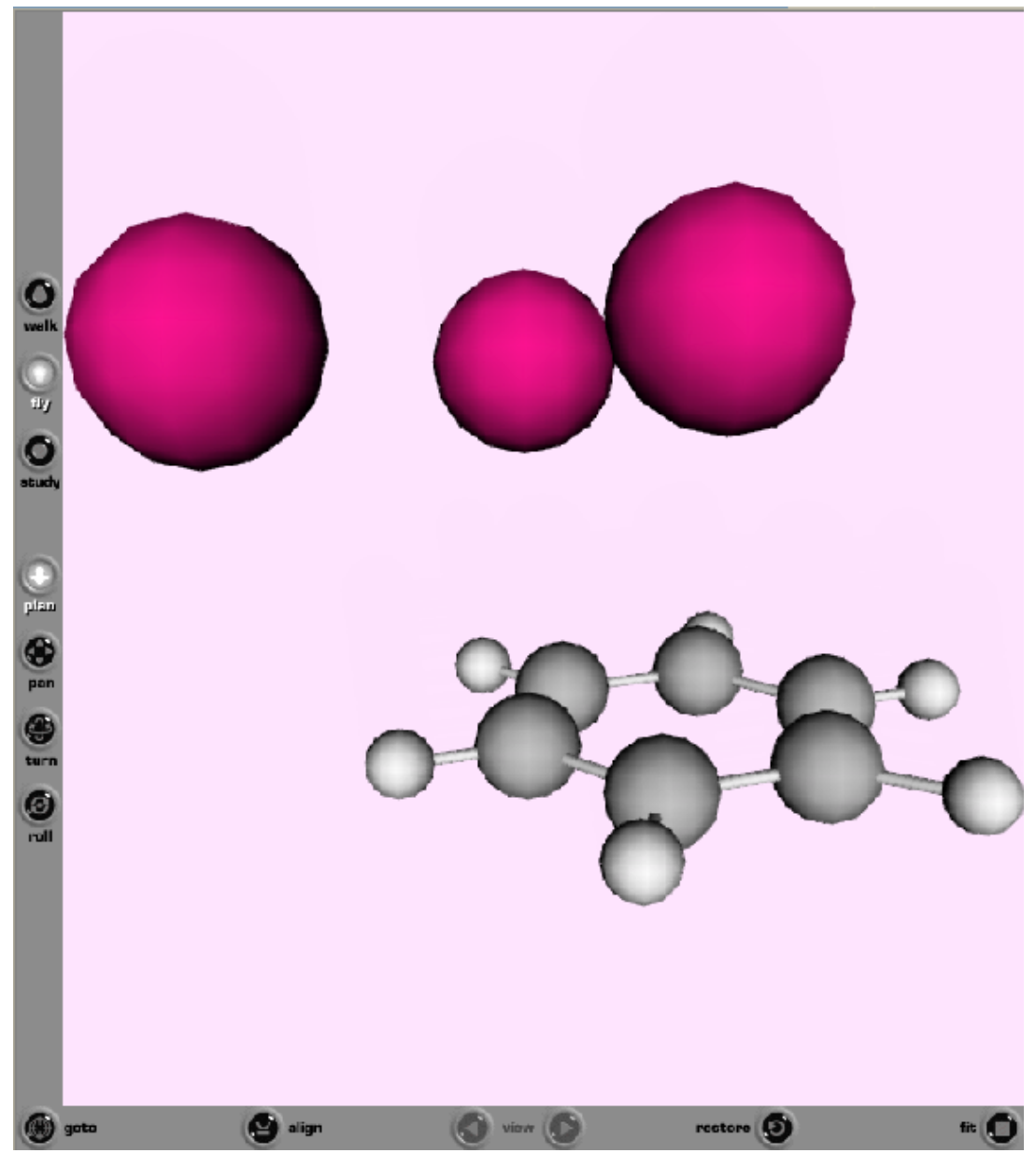

Fig. 2. An MVR representation of a typical benzene- $\mathrm{Ar}_{3}$ cluster

\section{The Molecular Dynamics Simulation}

As already mentioned, the MVR component of the chemical experiments considered in the present paper is based on the molecular dynamics suite of codes DL_POLY [21]. DL_POLY is organized as a distributable molecular dynamics simulation package of subroutines, programs and data files allowing the study of macromolecules, polymers, ionic systems and solutions. We use for our purposes its most recent version DL_POLY_2 (version 2.13). This version of DL_POLY includes a Graphical User Interface, written in Java, offering additional functionalities enhancing its usage in an MVR context. 
For illustrative purposes we discuss here the use of DL_POLY for the creation of an Ar seeded neutral benzene jet. This system is of particular interest since only a few benzene-rare gas $_{n}$ isomers can coexist during the lifetime of the cluster in the expansion jet. In connection with the relative abundances measured in the experiment, the dynamical simulation should predict structural transitions occurring during the cooling process.

Dynamical calculations are, as usual, carried out by expressing the interaction in terms of components having simple two-body well-known functional forms (like, for instance, the Lennard-Jones 12-6 or the Morse ones [22]). To overcome the limitations of the pairwise additive formulation of the potential that, as an example, makes it difficult to describe properly the interaction for geometries other than the most stable one, especially near the in-plane configurations [23], we have recently adopted also a new-semiempirical potential describing in a more realistic way the interaction between the benzene and the rare gas atoms [24].

Up to date, most of the runs have been carried out by considering clusters with two and three Ar atoms. A picture of a typical clustering situation for a three Ar atom system is illustrated in Fig. 2. The figure shows that a suitable geometry is that of three Ar atoms sitting on the same (upper, in the figure) side of the benzene molecule in a quite asymmetric configuration (this cannot be properly perceived in a single shot picture like Fig. 2 while it can be fully appreciated in an MVR environment in which the observer can move around the molecule).

\section{Conclusions}

The aim of the paper is to show how is possible to use virtual reality approaches to describe a chemical experiment at both a human and a molecular level using a virtual reality approach. To this end a multiscale virtual reality approach has been adopted to deal with the description of the physical environment, HVR scale, and with the molecular environment, MVR scale. The main features of the three experiments of different complexity and of the common molecular dynamics engine have been outlined in the paper. The application is still in a prototype stage. In spite of that, however, it has already shown to be very effective. This has prompted us to plan its porting on a grid platform in order to exploit its full potentiality.

\section{Acknowledgments}

This research has been financially supported by MIUR, CNR and ASI as well as by the D23 Action (Metachem) of COST in Chemistry. M.A. also acknowledges the financial support from the Ministerio de Educacion, Cultura y Deporte: PR2002-0014), the Spanish DGICYT (PB97-0919 and BQU2001-3018) and the Generalitat de Catalunya (CUR 20001SGR-00041). 


\section{References}

1. Wolff, R.S., Yaeger, L.: Visualization of Natural Phenomena. Springer-Verlag NewYork (1993)

2. Maldonado, T.: Reale e Virtuale. Feltrinelli, Milano (1994)

3. Zare, N.R.: Visualizing Chemistry. Journal of Chemical Education, Vol. 79 (2002) 1290-1291

4. Casher, O., Leach, C., Page, C.S., Rezpa, H.S.: Virtual reality modelling language (VRML) in Chemistry. Chemistry in Britain, Vol. 34 (1998) 26-31

5. Krieger, J.H: Doing Chemistry in a virtual world. Chemical \& Engineering News (1996) 35-41

6. Ruiz, I.R., Espinosa, E.L., Garcia, G.C., Gómez-Nieto, MÁ.: Computer-Assisted Learning of Chemical Experiments through a 3D Virtual Laboratory. Lecture Notes in Computer Science, Vol. 2329 (2002) 704-712

7. Garratt, J., Clow, D., Hodgson, A., and Tomlinson, A.: Computer Simulation and Chemical Education - A review of Project eLABorate. Chemistry Education Review, Vol. 14 (1999) 51-73 (ISSN 0972-0316).

8. Brattan, D. Jevons, O.M., and Rest, A.J. (Eds): Practical Laboratory Chemistry, Series of 19 CD ROM Programmes for teaching laboratory skills. Chemistry Video Consortium, Southampton, October 1999 (http://www.soton.ac.uk/ chemweb/cvc/ and http://www.emf-v.com).

9. http://vr.isdale.com/WhatIsVR.htm

10. Ames, A., L., Nadeau, D., R., Moreland, J., L.: VRML 2.0 Sourcebook. Wiley Computer Publishing, New York Tokio (1997)

11. Hartman, J., Wernecke, J.: VRML 2.0 Handbook: building moving worlds on the web. Addison Wesley (1996)

12. OpenSource cross-platform VRML browsers:

OpenVRML (http://www.openvrml.org); FreeWRL

(http://freewrl.sourceforge.net);

Vendor VRML browsers:

Cortona from Parallel Graphics (http://www.parallelgraphics.com/cortona/); Contact from Blaxxun (http://developer.blaxxun.com/); Cosmo Software from Computer Associates (http://www.cai.com/cosmo)

13. X3D Consortium: http://www.x3d.org

14. Flanagan, D.: Java in a Nutshell. O'Reilly (1999)

15. Chemistry Markup Language: http://www.xml-cml.org

16. EXtensible Markup Language: http://www.xml.org

17. Riganelli, A., Pacifici, L., Gervasi, O., Laganà, A., Workshop on Multimedia in Chemistry Education, Perugia, (2002)

18. http://www. chm.davidson.edu/ChemistryApplets/GasLaws/BoylesLaw.html

19. McKelvey, G.M., Gilbert, G.L., McWherter, C.: Flame Tests that Are Portable, Storable, and Easy to Use. Journal of Chemical Education, Vol. 75 (1998) 55-56

20. http://www. spectroscopynow. com

21. Smith, W., Forester, T.R., J., Molecular Graphics, Vol. 14 (1996) 136-141; http://www.dl.ac.uk/TCS/Software/DL POLY/

22. Stone, A.: The Theory of Intermolecular forces. Oxford University Press, Oxford (1996)

23. Amos, A.T., Palmer, T.F., Walters, A., Burrows, B.L., Chem. Phys. Letters, Vol. 172 (1990) 503-508

24. Pirani, F., Cappelletti, D., Liuti, G., Chem. Phys. Letters, Vol. 350 (2001) 286-289 